\title{
Computational Design and Experimental Synthesis of Air-stable Solid-state Ionic Conductors with High Conductivity
}

\author{
Ryounghee Kim ${ }^{1}$, Lincoln J. Miara ${ }^{2}$, Jung-Hwa Kim, ${ }^{3}{\mathrm{Ju}-\mathrm{Sik} \mathrm{Kim}^{1 *} \text {, Dongmin } \mathrm{Im}^{1} \text {, and Yan Wang }}^{2 *}$ \\ 1 Next Generation Battery Lab, Samsung Advanced Institute of Technology, Samsung Electronics Co., Ltd., Suwon- \\ si, Gyeonggi-do 16678, Republic of Korea \\ 2 Advanced Materials Lab, Samsung Advanced Institute of Technology-America, Samsung Semiconductor, Inc., \\ Cambridge, MA 02138, United States \\ 3 Autonomous Materials Development Lab, Samsung Advanced Institute of Technology, Samsung Electronics Co., \\ Ltd., Suwon-si, Gyeonggi-do 16678, Republic of Korea \\ Email: (Ju-Sik Kim) jusik.kim@samsung.com; (Yan Wang) eric.wangyan@samsung.com
}

\section{Supporting Information}


Table S1. DFT optimized crystal structure parameters of $\mathrm{Li}_{1+\mathrm{x}} \operatorname{Ta}_{2} \mathrm{P}_{1-\mathrm{x}} \mathrm{Si}_{\mathrm{x}} \mathrm{O}_{8}(x=0.125,0.25,0.375,0.5$, $0.625,0.75,0.875,1)$.

\begin{tabular}{|l|l|l|l|}
\hline Composition & a, b, c (angstrom) & $\begin{array}{l}\text { Unit-cell } \\
\text { Volume } \\
\text { (angstrom*3) }\end{array}$ & Density (gm/cc) \\
\hline $\mathbf{L i T a} \mathbf{P O}_{\mathbf{8}}$ & $9.86,11.74,10.92$ & 1263.49 & 5.55 \\
\hline $\mathbf{L i}_{\mathbf{1 . 1 2 5}} \mathbf{T a}_{\mathbf{2}} \mathbf{P}_{\mathbf{0 . 8 7 5}} \mathbf{S i}_{\mathbf{0 . 1 2 5}} \mathbf{O}_{\mathbf{8}}$ & $9.87,11.75,10.92$ & 1266.30 & 5.54 \\
\hline $\mathbf{L i}_{\mathbf{1 . 2 5}} \mathbf{T a}_{\mathbf{2}} \mathbf{P}_{\mathbf{0 . 7 5}} \mathbf{S i}_{\mathbf{0 . 2 5}} \mathbf{O}_{\mathbf{8}}$ & $9.88,11.74,10.88$ & 1262.18 & 5.57 \\
\hline $\mathbf{L i}_{\mathbf{1 . 3 7 5}} \mathbf{T a}_{\mathbf{2}} \mathbf{P}_{\mathbf{0 . 6 2 5}} \mathbf{S i}_{\mathbf{0 . 3 7 5}} \mathbf{O}_{\mathbf{8}}$ & $9.88,11.72,10.88$ & 1259.54 & 5.58 \\
\hline $\mathbf{L i}_{\mathbf{1 . 5}} \mathbf{T a}_{\mathbf{2}} \mathbf{P}_{\mathbf{0 . 5}} \mathbf{S i}_{\mathbf{0} \mathbf{5}} \mathbf{O}_{\mathbf{8}}$ & $9.88,11.73,10.87$ & 1258.78 & 5.59 \\
\hline $\mathbf{L i}_{\mathbf{1 . 6 2 5}} \mathbf{T a}_{\mathbf{2}} \mathbf{P}_{\mathbf{0 . 3 7 5}} \mathbf{S i}_{\mathbf{0 . 6 2 5}} \mathbf{O}_{\mathbf{8}}$ & $9.88,11.73,10.83$ & 1254.78 & 5.61 \\
\hline $\mathbf{L i}_{\mathbf{1 . 7 5}} \mathbf{T a}_{\mathbf{2}} \mathbf{P}_{\mathbf{0 . 2 5}} \mathbf{S i}_{\mathbf{0 . 7 5}} \mathbf{O}_{\mathbf{8}}$ & $9.88,11.73,10.79$ & 1251.09 & 5.64 \\
\hline $\mathbf{L i}_{\mathbf{1 . 8 7 5}} \mathbf{T a}_{\mathbf{2}} \mathbf{P}_{\mathbf{0 . 1 2 5}} \mathbf{S i}_{\mathbf{0} \mathbf{8 7 5}} \mathbf{O}_{\mathbf{8}}$ & $9.90,11.75,10.82$ & 1259.26 & 5.61 \\
\hline $\mathbf{L i}_{\mathbf{2}} \mathbf{T} \mathbf{a}_{\mathbf{2}} \mathbf{S i O}_{\mathbf{8}}$ & $9.90,11.74,10.78$ & 1252.14 & 5.64 \\
\hline
\end{tabular}



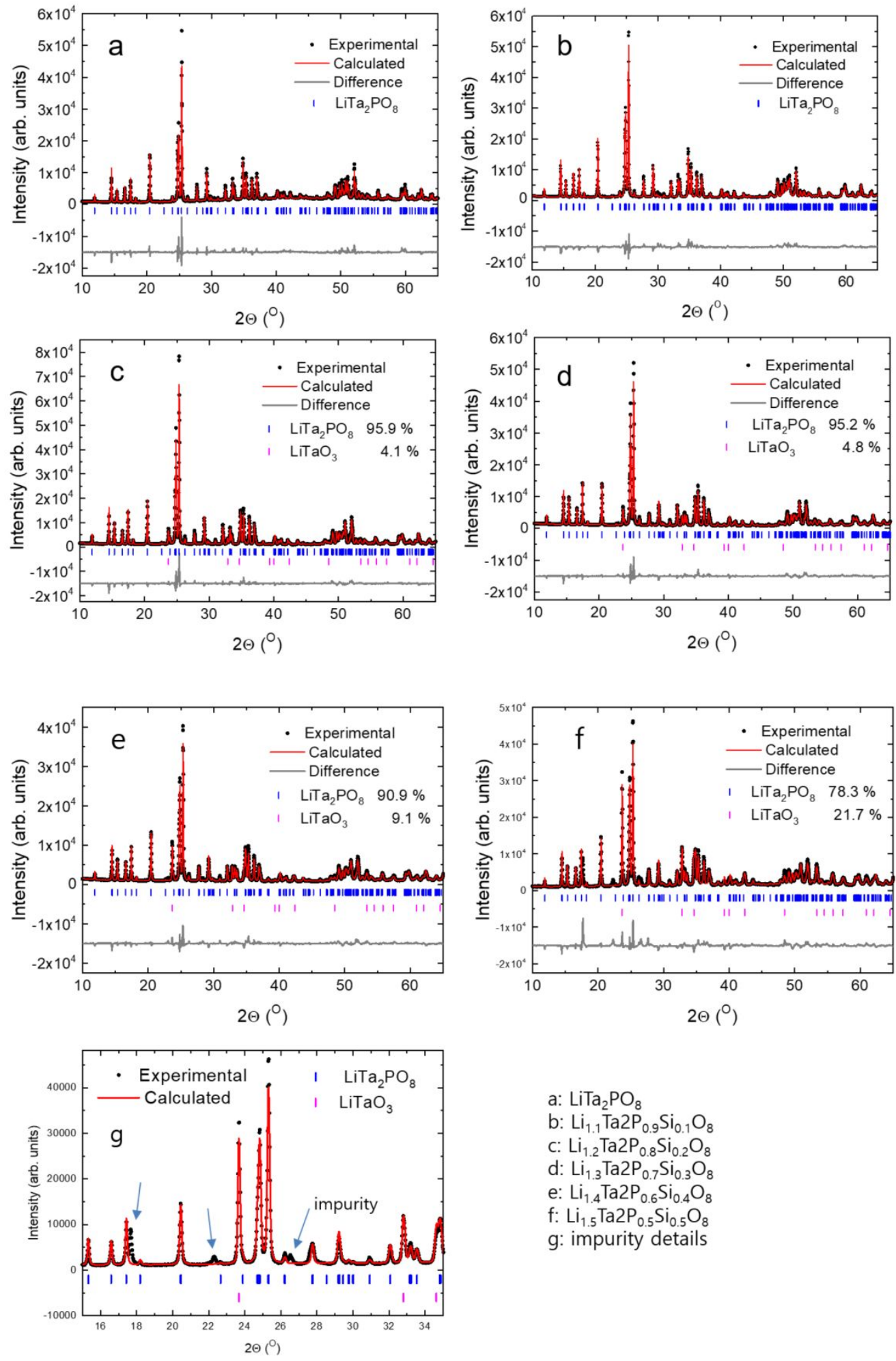

Figure S1. XRD pattern and Rietveld refinement of synthesized $\operatorname{Li}_{1+x} \operatorname{Ta}_{2} \mathrm{P}_{1-\mathrm{x}} \operatorname{Si}_{\mathrm{x}} \mathrm{O}_{8}(x=0,0.1,0.2,0.3$, 0.4, 0.5). 
Table S2. Refined crystal structure data including atom coordinates of $\mathrm{LiTa}_{2} \mathrm{PO}_{8}$ (space group $\mathrm{C2} / \mathrm{c})$. Rietveld refinements were performed using the structural model of $\mathrm{LiTa}_{2} \mathrm{PO}_{8}$ taken from the previous report. Since it is well known that XRD is not sensitive to light elements, the Li positions were fixed based on the pristine structure. The occupancies of $\mathrm{Li}, \mathrm{P}$ and $\mathrm{Si}$ were fixed to the values corresponding to the composition. The crystal structure of $\mathrm{LaTiO}_{3}$ was used which was reported at Golden Book of Phase Transitions, Wroclaw, 1, 1, (2002).

\begin{tabular}{|l|l|l|l|l|}
\hline Atom & X & y & Z & Occupancy \\
\hline Li1 & $0.245(0)$ & $0.357(0)(0)$ & $0.140(0)$ & $0.72(0)$ \\
\hline Li2 & $0.112(0)$ & 0.387 & $0.4(0)$ & $0.21(0)$ \\
\hline Li3 & $0.5(0)$ & 0 & 0 & $0.14(0)$ \\
\hline Ta1 & $0.248(1)$ & $0.097(1)$ & $0.255(1)$ & $1(0)$ \\
\hline Ta2 & 0 & $0.346(1)$ & $0 z .25(0)$ & $1(0)$ \\
\hline Ta3 & 0 & 0 & 0 & $1(0)$ \\
\hline P1 & $0.489(5)$ & $0.208(2)$ & $0.060(2)$ & $1(0)$ \\
\hline O1 & $0.058(5)$ & $0.335(4)$ & $0.066(5)$ & $1(0)$ \\
\hline O2 & $0.382(3)$ & $0.140(3)$ & $0.413(4)$ & $1(0)$ \\
\hline O3 & $0.359(3)$ & $0.496(3)$ & $0.125(3)$ & $1(0)$ \\
\hline O4 & $0.047(4)$ & $0.168(3)$ & $0.561(4)$ & $1(0)$ \\
\hline O5 & $0.147(3)$ & $0.239(2)$ & $0.294(4)$ & $1(0)$ \\
\hline O6 & $0.141(6)$ & $0.056(7)$ & $0.114(8)$ & $1(0)$ \\
\hline O7 & $0.386(3)$ & $0.206(3)$ & $0.154(4)$ & $1(0)$ \\
\hline O8 & $0.135(3)$ & $0.469(3)$ & $0.288(6)$ & $1(0)$ \\
\hline
\end{tabular}


Table S3. Refined crystal structure data including atom coordinates of $\operatorname{Li}_{1.1} \operatorname{Ta}_{2} \mathrm{P}_{0.9} \mathrm{Si}_{0.1} \mathrm{O}_{8}(\mathrm{Space}$ group $\mathrm{C2} / \mathrm{c}$ ).

\begin{tabular}{|l|l|l|l|l|}
\hline Atom & $\mathrm{x}$ & $\mathrm{y}$ & $\mathrm{z}$ & Occupancy \\
\hline Li1 & $0.245(0)$ & $0.357(0)(0)$ & $0.140(0)$ & $0.77(0)$ \\
\hline Li2 & $0.112(0)$ & 0.387 & $0.4(0)$ & $0.26(0)$ \\
\hline Li3 & $0.5(0)$ & 0 & 0 & $0.14(0)$ \\
\hline Ta1 & $0.246(1)$ & $0.098(1)$ & $0.254(1)$ & $1(0)$ \\
\hline Ta2 & 0 & $0.349(1)$ & $0.25(0)$ & $1(0)$ \\
\hline Ta3 & 0 & 0 & 0 & $1(0)$ \\
\hline P1 & $0.489(2)$ & $0.206(1)$ & $0.059(1)$ & $0.9(0)$ \\
\hline Si1 & $0.489(2)$ & $0.206(1)$ & $0.059(1)$ & $0.1(0)$ \\
\hline O1 & $0.056(3)$ & $0.335(2)$ & $0.066(2)$ & $1(0)$ \\
\hline O2 & $0.383(2)$ & $0.140(2)$ & $0.414(2)$ & $1(0)$ \\
\hline O3 & $0.359(4)$ & $0.496(4)$ & $0.125(5)$ & $1(0)$ \\
\hline O4 & $0.047(3)$ & $0.168(2)$ & $0.561(2)$ & $1(0)$ \\
\hline O5 & $0.147(2)$ & $0.237(2)$ & $0.288(3)$ & $1(0)$ \\
\hline O6 & $0.141(2)$ & $0.056(2)$ & $0.114(2)$ & $1(0)$ \\
\hline O7 & $0.386(2)$ & $0.206(2)$ & $0.153(2)$ & $1(0)$ \\
\hline O8 & $0.134(2)$ & $0.467(1)$ & $0.288(3)$ & $1(0)$ \\
\hline
\end{tabular}

Table S4. Refined crystal structure data including atom coordinates of $\operatorname{Li}_{1.2} \operatorname{Ta}_{2} \mathbf{P}_{0.8} \mathrm{Si}_{0.2} \mathrm{O}_{8}(\mathrm{Space}$ group $\mathrm{C2} / \mathrm{c}$ ).

\begin{tabular}{|l|l|l|l|l|}
\hline Atom & $\mathrm{x}$ & $\mathrm{y}$ & $\mathrm{z}$ & Occupancy \\
\hline Li1 & $0.245(0)$ & $0.357(0)(0)$ & $0.140(0)$ & $0.82(0)$ \\
\hline Li2 & $0.112(0)$ & 0.387 & $0.4(0)$ & $0.31(0)$ \\
\hline Li3 & $0.5(0)$ & 0 & 0 & $0.14(0)$ \\
\hline Ta1 & $0.247(1)$ & $0.098(1)$ & $0.256(1)$ & $1(0)$ \\
\hline
\end{tabular}




\begin{tabular}{|l|l|l|l|l|}
\hline Ta2 & 0 & $0.350(1)$ & $0.25(0)$ & $1(0)$ \\
\hline Ta3 & 0 & 0 & 0 & $1(0)$ \\
\hline P1 & $0.496(3)$ & $0.206(1)$ & $0.061(1)$ & $0.8(0)$ \\
\hline Si1 & $0.496(3)$ & $0.206(1)$ & $0.061(1)$ & $0.2(0)$ \\
\hline O1 & $0.056(3)$ & $0.335(2)$ & $0.066(3)$ & $1(0)$ \\
\hline O2 & $0.379(2)$ & $0.136(2)$ & $0.412(2)$ & $1(0)$ \\
\hline O3 & $0.362(2)$ & $0.516(1)$ & $0.125(2)$ & $1(0)$ \\
\hline O4 & $0.048(2)$ & $0.168(2)$ & $0.561(2)$ & $1(0)$ \\
\hline O5 & $0.147(1)$ & $0.239(1)$ & $0.287(3)$ & $1(0)$ \\
\hline O6 & $0.141(3)$ & $0.056(4)$ & $0.114(5)$ & $1(0)$ \\
\hline O7 & $0.383(2)$ & $0.206(1)$ & $0.153(2)$ & $1(0)$ \\
\hline O8 & $0.134(2)$ & $0.46(2)$ & $0.288(4)$ & $1(0)$ \\
\hline
\end{tabular}

Table S5. Refined crystal structure data including atom coordinates of $\mathrm{Li}_{1.3} \mathrm{Ta}_{2} \mathbf{P}_{0.7} \mathrm{Si}_{0.3} \mathrm{O}_{8}(\mathrm{Space}$ group C2/c).

\begin{tabular}{|l|l|l|l|l|}
\hline Atom & X & y & Z & Occupancy \\
\hline Li1 & $0.245(0)$ & $0.357(0)(0)$ & $0.140(0)$ & $0.87(0)$ \\
\hline Li2 & $0.112(0)$ & 0.387 & $0.4(0)$ & $0.36(0)$ \\
\hline Li3 & $0.5(0)$ & 0 & 0 & $0.14(0)$ \\
\hline Ta1 & $0.246(1)$ & $0.098(1)$ & $0.255(1)$ & $1(0)$ \\
\hline Ta2 & 0 & $0.351(1)$ & $0.25(0)$ & $1(0)$ \\
\hline Ta3 & 0 & 0 & 0 & $1(0)$ \\
\hline P1 & $0.498(3)$ & $0.209(1)$ & $0.061(1)$ & $0.7(0)$ \\
\hline Si1 & $0.498(3)$ & $0.209(1)$ & $0.061(1)$ & $0.3(0)$ \\
\hline O1 & $0.056(4)$ & $0.328(2)$ & $0.066(3)$ & $1(0)$ \\
\hline O2 & $0.379(3)$ & $0.135(2)$ & $0.406(3)$ & $1(0)$ \\
\hline
\end{tabular}




\begin{tabular}{|l|l|l|l|l|}
\hline O3 & $0.365(2)$ & $0.516(1)$ & $0.125(2)$ & $1(0)$ \\
\hline O4 & $0.048(3)$ & $0.168(2)$ & $0.561(2)$ & $1(0)$ \\
\hline O5 & $0.147(2)$ & $0.237(1)$ & $0.285(3)$ & $1(0)$ \\
\hline O6 & $0.141(4)$ & $0.057(3)$ & $0.114(6)$ & $1(0)$ \\
\hline O7 & $0.376(2)$ & $0.207(1)$ & $0.157(3)$ & $1(0)$ \\
\hline O8 & $0.134(2)$ & $0.46(2)$ & $0.288(4)$ & $1(0)$ \\
\hline
\end{tabular}

Table S6. Refined crystal structure data including atom coordinates of $\operatorname{Li}_{1.4} \mathrm{Ta}_{2} \mathbf{P}_{0.6} \mathrm{Si}_{0.4} \mathbf{O}_{8}$ (Space group C2/c).

\begin{tabular}{|l|l|l|l|l|}
\hline Atom & X & y & $z$ & Occupancy \\
\hline Li1 & $0.245(0)$ & $0.357(0)(0)$ & $0.140(0)$ & $0.92(0)$ \\
\hline Li2 & $0.112(0)$ & 0.387 & $0.4(0)$ & $0.41(0)$ \\
\hline Li3 & $0.5(0)$ & 0 & 0 & $0.14(0)$ \\
\hline Ta1 & $0.247(1)$ & $0.098(1)$ & $0.255(1)$ & $1(0)$ \\
\hline Ta2 & 0 & $0.353(1)$ & $0.25(0)$ & $1(0)$ \\
\hline Ta3 & 0 & 0 & 0 & $1(0)$ \\
\hline P1 & $0.494(6)$ & $0.206(1)$ & $0.061(2)$ & $0.6(0)$ \\
\hline Si1 & $0.494(6)$ & $0.206(1)$ & $0.061(2)$ & $0.4(0)$ \\
\hline O1 & $0.058(5)$ & $0.333(3)$ & $0.066(5)$ & $1(0)$ \\
\hline O2 & $0.375(4)$ & $0.135(3)$ & $0.414(4)$ & $1(0)$ \\
\hline O3 & $0.365(3)$ & $0.516(2)$ & $0.125(3)$ & $1(0)$ \\
\hline O4 & $0.048(5)$ & $0.166(3)$ & $0.561(4)$ & $1(0)$ \\
\hline O5 & $0.147(3)$ & $0.237(2)$ & $0.284(4)$ & $1(0)$ \\
\hline O6 & $0.141(7)$ & $0.058(6)$ & $0.114(8)$ & $1(0)$ \\
\hline O7 & $0.371(4)$ & $0.205(3)$ & $0.157(4)$ & $1(0)$ \\
\hline O8 & $0.134(4)$ & $0.459(3)$ & $0.288(7)$ & $1(0)$ \\
\hline
\end{tabular}


Table S7. Refined crystal structure data including atom coordinates of $\mathrm{Li}_{1.5} \operatorname{Ta}_{2} \mathrm{P}_{0.5} \mathrm{Si}_{0.5} \mathrm{O}_{8}(\mathrm{Space}$ group $\mathrm{C2} / \mathrm{c}$ ).

\begin{tabular}{|l|l|l|l|l|}
\hline Atom & $\mathrm{x}$ & $\mathrm{y}$ & $\mathrm{z}$ & Occupancy \\
\hline Li1 & $0.245(0)$ & $0.357(0)(0)$ & $0.140(0)$ & $0.97(0)$ \\
\hline Li2 & $0.112(0)$ & 0.387 & $0.4(0)$ & $0.46(0)$ \\
\hline Li3 & $0.5(0)$ & 0 & 0 & $0.14(0)$ \\
\hline Ta1 & $0.246(1)$ & $0.098(1)$ & $0.256(1)$ & $1(0)$ \\
\hline Ta2 & 0 & $0.353(1)$ & $0.25(0)$ & $1(0)$ \\
\hline Ta3 & 0 & 0 & 0 & $1(0)$ \\
\hline P1 & $0.489(8)$ & $0.205(3)$ & $0.061(3)$ & $0.5(0)$ \\
\hline Si1 & $0.489(8)$ & $0.205(3)$ & $0.061(3)$ & $0.5(0)$ \\
\hline O1 & $0.058(1)$ & $0.341(6)$ & $0.066(9)$ & $1(0)$ \\
\hline O2 & $0.374(7)$ & $0.134(6)$ & $0.399(7)$ & $1(0)$ \\
\hline O3 & $0.365(6)$ & $0.517(5)$ & $0.125(6)$ & $1(0)$ \\
\hline O4 & $0.048(9)$ & $0.168(6)$ & $0.561(7)$ & $1(0)$ \\
\hline O5 & $0.147(6)$ & $0.239(4)$ & $0.284(9)$ & $1(0)$ \\
\hline O6 & $0.141(12)$ & $0.057(11)$ & $0.114(15)$ & $1(0)$ \\
\hline O7 & $0.371(8)$ & $0.206(5)$ & $0.157(8)$ & $1(0)$ \\
\hline O8 & $0.134(7)$ & $0.461(5)$ & $0.288(13)$ & $1(0)$ \\
\hline
\end{tabular}




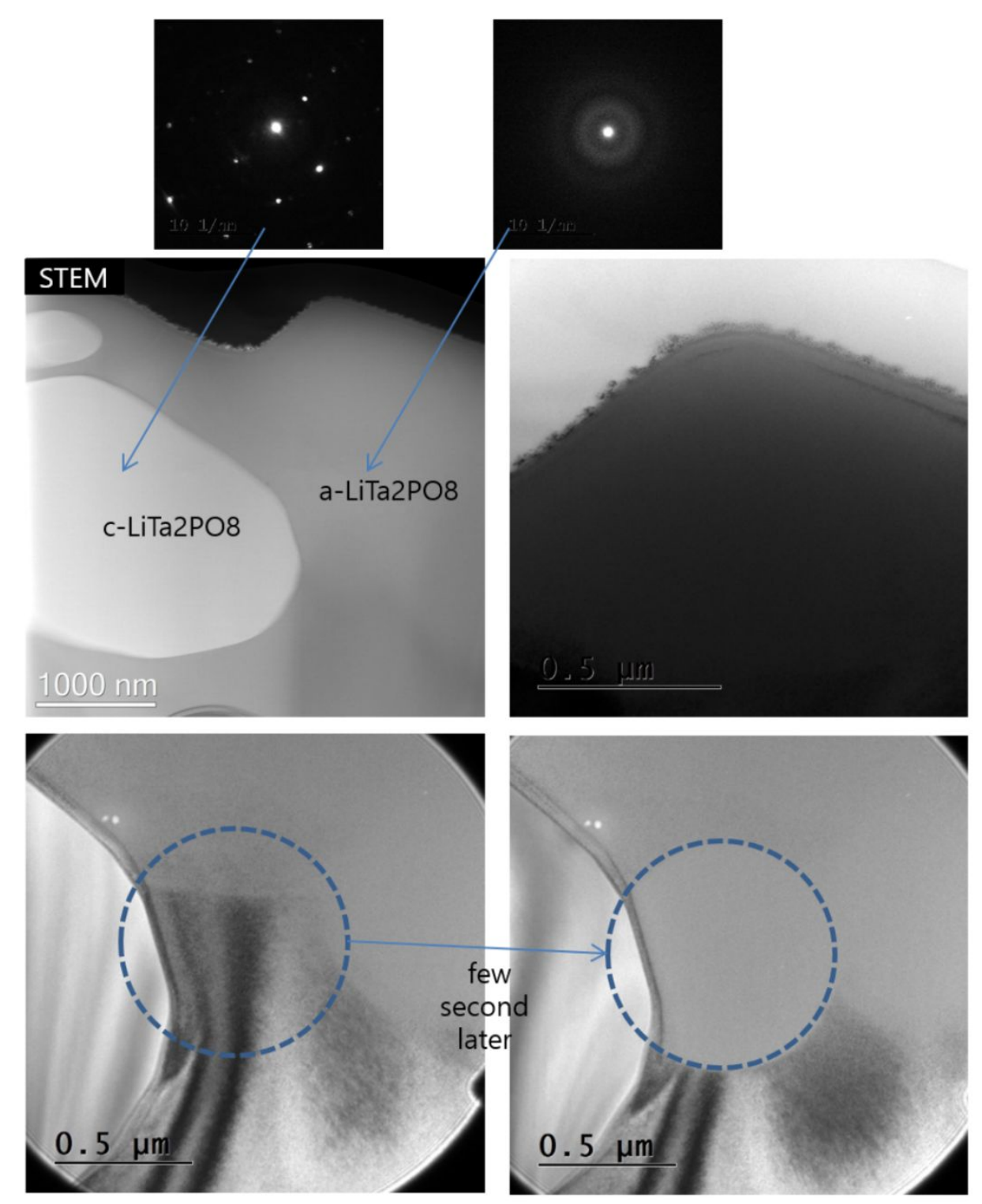

Figure S2. STEM images of $\mathrm{LiTa}_{2} \mathrm{PO}_{8}$ (Calcined powder), showing that the sample contain amorphous regions, and these phases tend to be easily damaged by the radiolysis damage caused by electronic excitation or ionization from the electron beam. 

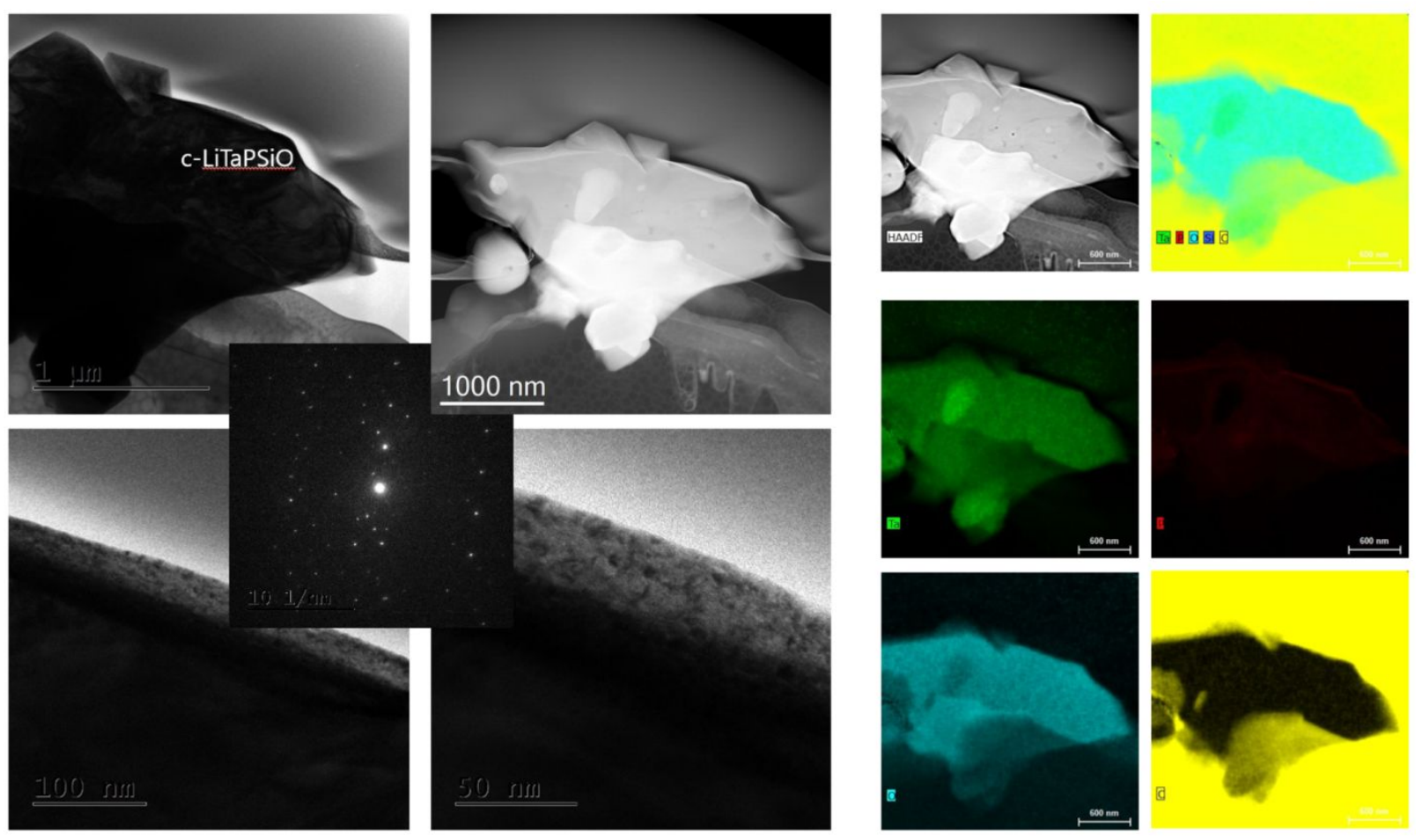

Figure S3. STEM images of $\mathrm{Li}_{1.4} \mathrm{Ta}_{2} \mathrm{P}_{0.6} \mathrm{Si}_{0.4} \mathrm{O}_{8}$ (Calcined powder), showing the sample is mostly crystalline, and maintains the crystalline phase after exposure of electron beam. 


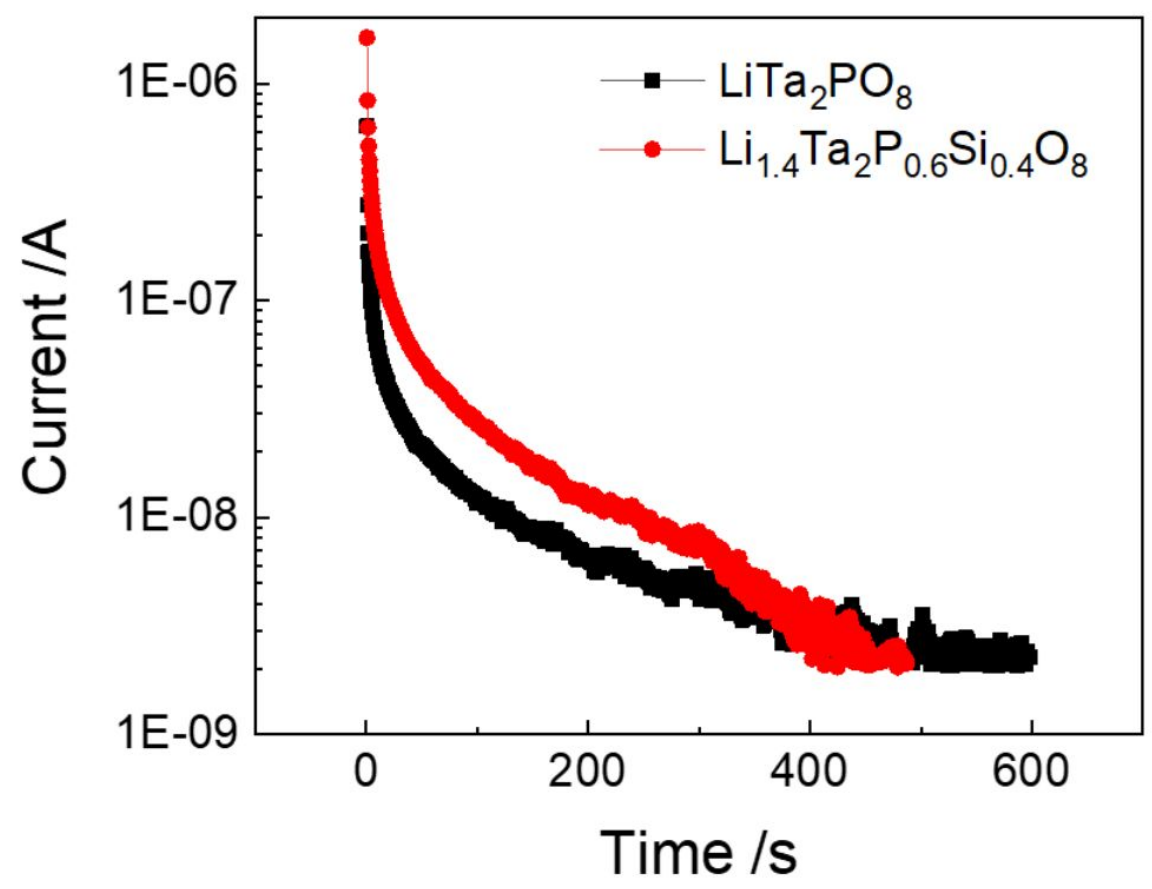

Fig. S4. D. C. polarization curves of LTPO (black) and LTPSO (Red). 


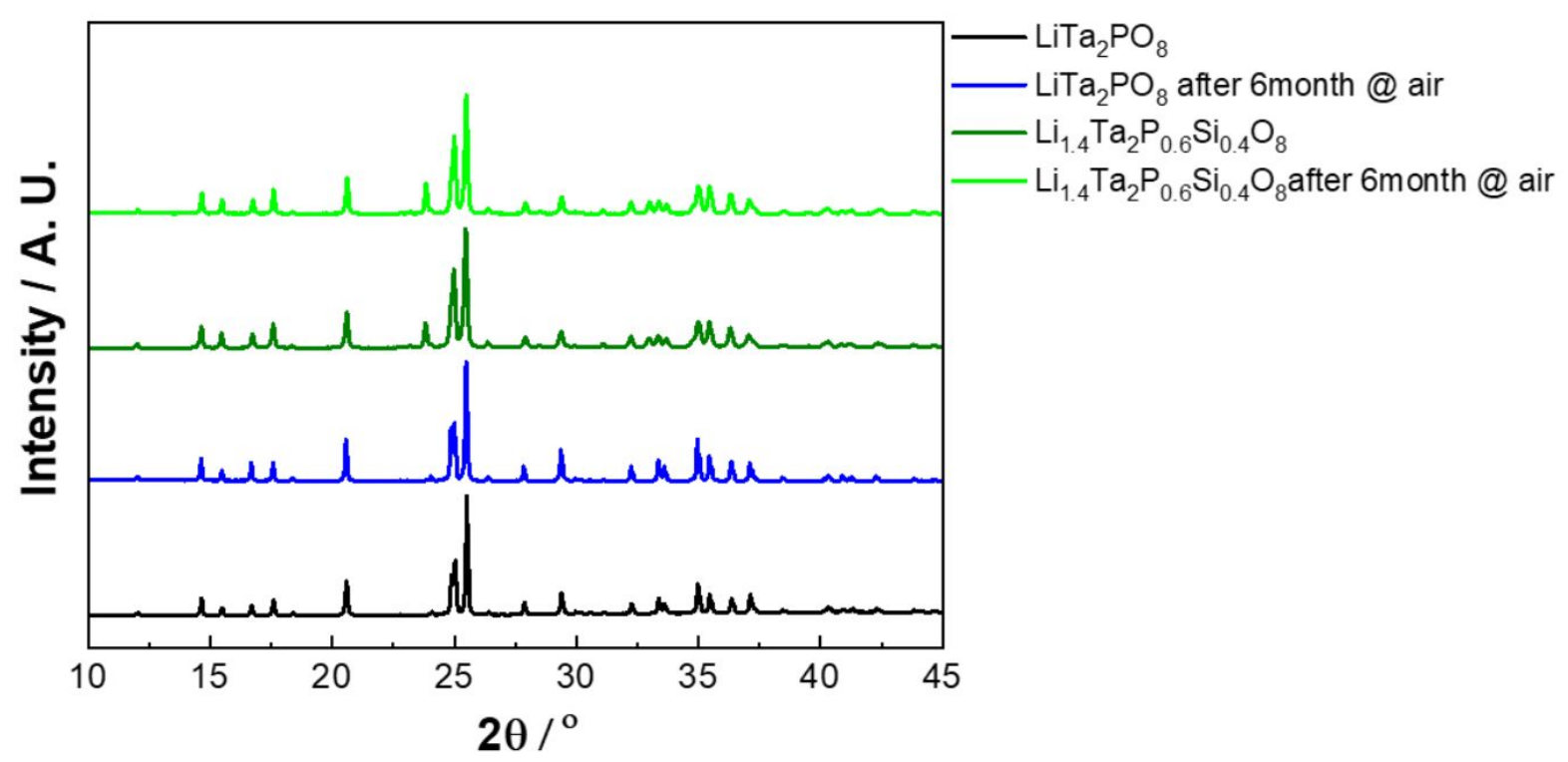

Figure S5. XRD pattern of LTPO and LTPSO samples measured after 6 months of ambient exposure after synthesis. 


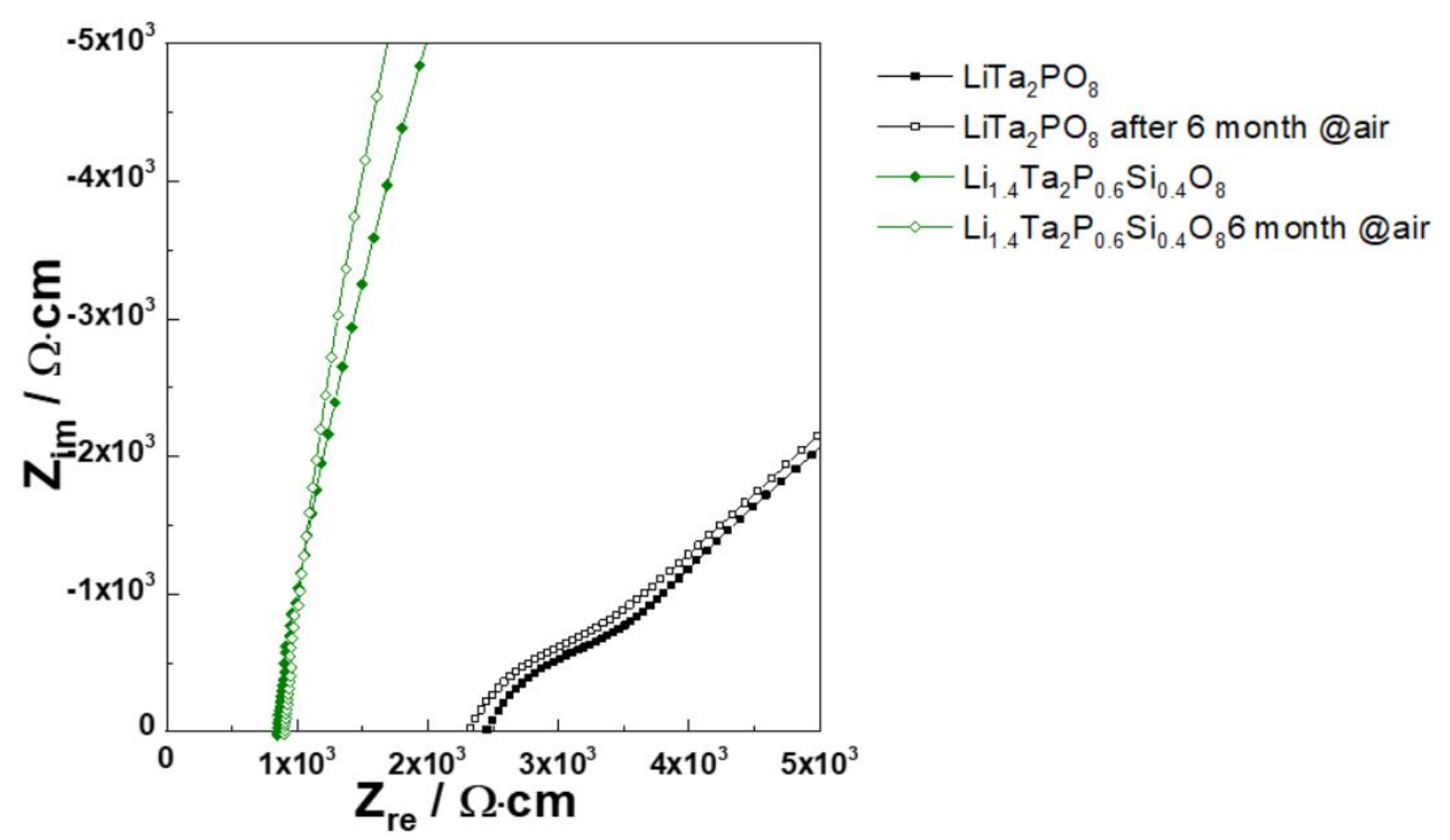

Figure S6. Impedance measurement of LTPO and LTPSO samples performed after 6 months of ambient exposure after synthesis 